1 THE EFFECT OF FASCIOTOMY FOR THE TREATMENT OF CHRONIC EXERTIONAL COMPARTMENT SYNDROME OF THE LOWER LEG

${ }^{1}$ Nicolai Sandau*, ${ }^{2}$ Kasper Guldbrandsen, ${ }^{2}$ Francesca Cucchi, ${ }^{1}$ Cristina Oprea, ${ }^{2}$ Lars Friberg, ${ }^{1}$ Lars Konradsen. ${ }^{1}$ Department of Orthopedic Surgery - Section for Sports Traumatology, Bispebjerg-Frederiksberg Hospital, University of Copenhagen Part of IOC Research Center Copenhagen, Denmark; ${ }^{2}$ Department of Clinical Physiology and Nuclear Medicine, Bispebjerg-Frederiksberg Hospital, Denmark

\subsection{6/bjsports-2019-scandinavianabs.1}

Introduction This study aims to report the post-surgical objective functional outcome in patients with chronic exertional compartment syndrome (CECS) having undergone primary fasciotomy.

Materials and methods Patients who underwent fasciotomy for CECS at our facility between July 2014 and December 2017 with pre-operative data were retrospectively identified. Preoperatively the patients underwent a treadmill test and the time until they had to stop due to symptoms and/or experienced pain equal to an $8-10$ on the VAS scale was recorded. At a minimum of 8 months after surgery the treadmill test was performed again at the same speed and inclination as the pre-surgery test. Pain was then recorded at the exact time they either had to stop or experienced a pain of $8-10$ on the vas scale during the pre-surgery test.

Results A total of 19 patients were included, with 8 patients ( $5 \mathrm{~F} 3 \mathrm{M}$ ) in the anterior fasciotomy group (median age 24.73 years) and 11 patients $(9 \mathrm{~F} 3 \mathrm{M}$ ) in the posterior fasciotomy group (median age 22.78 years). The anterior fasciotomy group experienced a decrease in VAS from a median of 8 pre-operatively to 1.5 post-operatively $(\mathrm{p}<0.01)$. The posterior fasciotomy group experienced a decrease in VAS from a median of 8.25 pre-operatively to 4 post-operatively $(p<0.01)$. Conclusion Fasciotomy significantly decreased pain during activity in patients with anterior and posterior CECS. To our knowledge this is the first study to report an objective measure of the functional outcome following primary fasciotomy for the treatment of CECS.

\section{NON-UNIFORMITY IN PRE-INSERTIONAL ACHILLES TENDON IS NOT INFLUENCED BY CHANGING KNEE ANGLE DURING ISOMETRIC CONTRACTIONS}

${ }^{1}$ Stijn Bogaerts*, ${ }^{2,3}$ Catarina De Brito Carvalho, ${ }^{1,4}$ An De Groef, ${ }^{1}$ Koen Peers. ${ }^{1}$ Dept. of Development and Regeneration, KU Leuven/Dept. of Physical Medicine and Rehabilitation, University Hospitals Leuven, Belgium; ${ }^{2}$ ESAT/PSI and UZ Leuven, MIRC, KU Leuven and University Hospitals Leuven, Belgium; ${ }^{3}$ INESC TEC Porto, Instituto de Engenharia de Sistemas e Computadores - Tecnologia e Ciência, Portugal; ${ }^{4}$ Department of Rehabilitation Sciences, KU Leuven, Belgium

10.1136/bjsports-2019-scandinavianabs.2

Introduction Mechanical loading in Achilles tendinopathy, e.g. eccentric exercise, is commonly performed in both extended and flexed knee positions. The goal of this study was to evaluate the impact of this changing knee angle on the non-uniform behaviour in the Achilles tendon, i.e. superficial-to-deep variation in displacement with highest displacement in the deep layer. It was hypothesized that a flexed knee position would lead to a more non-uniform behaviour, due to greater differential loading of soleus versus gastrocnemius in this position.
Materials and methods Nineteen healthy subjects participated in this study. A high-spatial and high-temporal resolution US system was used to acquire $2 \mathrm{D}+$ time ultrasound images during an isometric contraction at 25\%,50\% and 75\% MVC in the extended and flexed knee position. Local tendon tissue displacement of the different tendon layers and normalized displacement ratio, a measure for non-uniformity, were calculated.

Results Contrary to the hypothesis, it was found that the nonuniform deformation, with highest displacement in the deep tendon layer, is consistently present and not influenced by a change in knee angle.

Conclusion From clinical perspective, this might indicate the absence of a mechanical rationale for a change in knee angle during loading exercises. Additionally, it was found that despite reaching high levels of force, the contribution of the AT to this deformation is sometimes unexpectedly smaller, potentially due to compensatory actions. This is relevant for AT rehabilitation as the goal there is to reach specific local tendon loading.

\section{LOW-LOAD RESISTANCE EXERCISE, BLOOD FLOW RESTRICTION, OR SHAM BLOOD FLOW RESTRICTION FOR ANTERIOR KNEE PAIN. A THREE-ARM PILOT RCT}

Vasileios Korakakis*, Rodney Whiteley. Aspetar, Sports Medicine Hospital, Qatar

\subsection{6/bjsports-2019-scandinavianabs.3}

Introduction Blood flow restriction (BFR) with low-load resistance exercise (LLRE) can induce significant pain reduction in anterior knee pain (AKP). This study evaluated the BFR-LLRE effect on pain compared to sham-BFR, or LLRE alone in patients with AKP.

Materials and methods 36 patients were randomly allocated to BFR, sham-BFR, or control group in this single-blinded trial. Pain was assessed immediately after intervention, and after a sequential physiotherapy session (45 min). BFR pressure was set to $80 \%$ of complete occlusion. All groups performed four sets of knee extensions with individualized load $(\leq 5 \mathrm{~kg})$ as indicated by a pain monitoring approach (pain $\leq 4 / 10$ ). Outcome measures were pain (0-10) during shallow and deep single-leg squat (SLSs-SLSd), and step-down test (SDT). Data were analyzed using factorial repeated measures ANOVA and were interpreted as clinically meaningful changes $(\mathrm{d}>0.80$; $30 \%$ pain reduction) and numbers needed to treat (NNT).

Results Groups did not differ at baseline. No significant between-group differences were found.

Significant within-group immediate pain reductions found only in BFR group ( $\leq \leq 0.003 ;$ SLSs $/ \mathrm{d}=1.44, \quad \mathrm{SLSd} / \mathrm{d}=1.27$, $\mathrm{SDT} / \mathrm{d}=0.94$ ), but not in sham-BFR and control group (all $\mathrm{p}>0.05)$.

Following physiotherapy session, pain was sustained significantly reduced only in BFR group $(\mathrm{p} \leq 0.003 ;$ SLS $\mathrm{s} / \mathrm{d}=1.71$, $\mathrm{SLSd} / \mathrm{d}=0.97, \mathrm{SDT} / \mathrm{d}=0.84)$, but not in sham-BFR and control group (all $\mathrm{p}>0.05$ ).

NNT ranged from 2 to 4 depending on the outcome measure used.

Conclusion BFR reduces pain independent of the effect of exercise. One BFR-exercise bout immediately reduced AKP with the effect sustained for at least $45 \mathrm{~min}$, an effect not seen with sham-BFR and without BFR. 